\title{
Open the gates: vascular neurocrine signaling mobilizes hematopoietic stem and progenitor cells
}

\author{
Tomer Itkin, Jesús María Gómez-Salinero, and Shahin Rafii \\ Division of Regenerative Medicine, Ansary Stem Cell Institute, Department of Medicine, Weill Cornell Medicine, New York, New York, USA.
}

\begin{abstract}
Mobilization of hematopoietic stem and progenitor cells (HSPCs) from the bone marrow (BM) into the peripheral blood is a complex process that is enhanced dramatically under stress-induced conditions. A better understanding of how the mobilization process is regulated will likely facilitate the development of improved clinical protocols for stem cell harvesting and transplantation. In this issue of the JCI, Singh et al. (1) showed that the truncated cleaved form of neurotransmitter neuropeptide $\mathrm{Y}$ (NPY) actively promotes a breach of BM vascular sinusoidal portals, thereby augmenting HSPC trafficking to the circulation. The authors report a previously unrecognized axis, in which expression of the enzyme dipeptidylpeptidase-4 (DPP4)/CD26 by endothelial cells activates NPYmediated signaling by increasing the bioavailability of the truncated form of NPY. These findings underscore the importance of and urgency to develop pharmacological therapies that target the vasculature and regulate diverse aspects of hematopoiesis, such as HSPC trafficking, in steady-state and stress-induced conditions.
\end{abstract}

\section{Overview of clinical}

hematopoietic stem and progenitor cell mobilization

The ability of hematopoietic stem and progenitor cells (HSPCs) to mobilize from the bone marrow (BM) is essential for the treatment of patients with hematological malignancies or disorders who undergo curative BM transplantation. For the past few decades, granulocyte CSF (G-CSF) has been the preferred mobilizing agent in clinical transplantation protocols (Figure 1); however, it is not fully clear how G-CSF induces stem cell mobilization or why certain individuals are resistant to G-CSF-mediated mobilization (2). Notably, approximately $10 \%$ of allogeneic donors and $40 \%$ of autologous donors fail to mobilize sufficient numbers of BM HSPCs to establish long-term engraftment (3), often resulting in aborted curative transplantation (4). Uncovering the pathways that modulate the mobilization of HSPCs could potentially offer a life-saving approach for the patients in need of a BM graft.

In response to G-CSF stimulation, recruitment of the CXCL12/CXCR4 signaling axis is one of the first steps for enhanced HSPC proliferation, motility, and egress into the peripheral blood $(2,5)$. Following G-CSF infusion, CXCL12 levels drop dramatically in BM (6) but increase in the circulation (7), possibly via endothelial re-secretion of scavenged CXCL12 (ref. 8 and Figure 1). Additionally, CXCR4 is upregulated by G-CSF in both murine and human HSPCs (6), further supporting the importance of the CXCL12/CXCR4 axis in HSPC mobilization. Notably, following circadian epinephrine secretion by the sympathetic nervous system, $\mathrm{CXCl} 12$ levels oscillate within the BM microenvironment, orchestrating steady-state egress

Related Article: p. 4527

(9). These findings highlight a regulatory role of the nervous system in the G-CSFinduced mobilization process.

Indeed, the sympathetic nervous system is pivotal for G-CSF-induced mobilization, as immature HSPCs express both dopaminergic and adrenergic receptors (10), which are further upregulated during G-CSFinduced mobilization. Moreover, mice with aberrant sympathetic nerve conduction are unable to downregulate CXCL12 in the BM and consequently fail to mobilize HSPCs in response to G-CSF (11). G-CSF treatment suppresses norepinephrine reuptake, thereby enhancing mobilized HSPCs (12). Therefore, oscillatory inputs from the nervous system during physiological HSPC trafficking and stressed-induced mobilization modulate BM cytokines and other putative proteolytic enzymes, thereby masterminding a balanced mobilization of HSPCs to the peripheral circulation (Figure 1).

Proteolytic activation of several factors is important during HSPC mobilization. Of note, CXCL12 mediates the release of soluble cleaved stem cell factor (SCF) via activation of MMP-9, resulting in enhanced HSPC egress from the BM (13). The membrane-bound type 1 MMP (MT1-MMP) is also expressed by HSPCs, upregulated following G-CSF stimulation, and actively participates in the HSPC mobilization process by cleaving the membrane-associated CD44 homing adhesion molecule (14). In addition, osteoclast-secreted cathepsin $\mathrm{K}$ (15) and the dipeptidylpeptidase 4 (DPP4, also known and herein referred to as CD26) (16) both cleave CXCL12 in vitro, which in turn may promote the degradation of CXCL12 in vivo. These data indicated a previously unappreciated role for proteolytic factors such as CD26 in HSPC mobilization.

Remarkably, G-CSF-induced mobilization of murine HSPCs is hampered by CD26 inhibition or in mice lacking $C D 26(16,17)$. CD26 cleaves a variety of proteins such as GM-CSF, G-CSF, IL-3, and erythropoietin that belong to the hematopoietic CSF family (18), deactivating further hematopoietic 
A Steady state

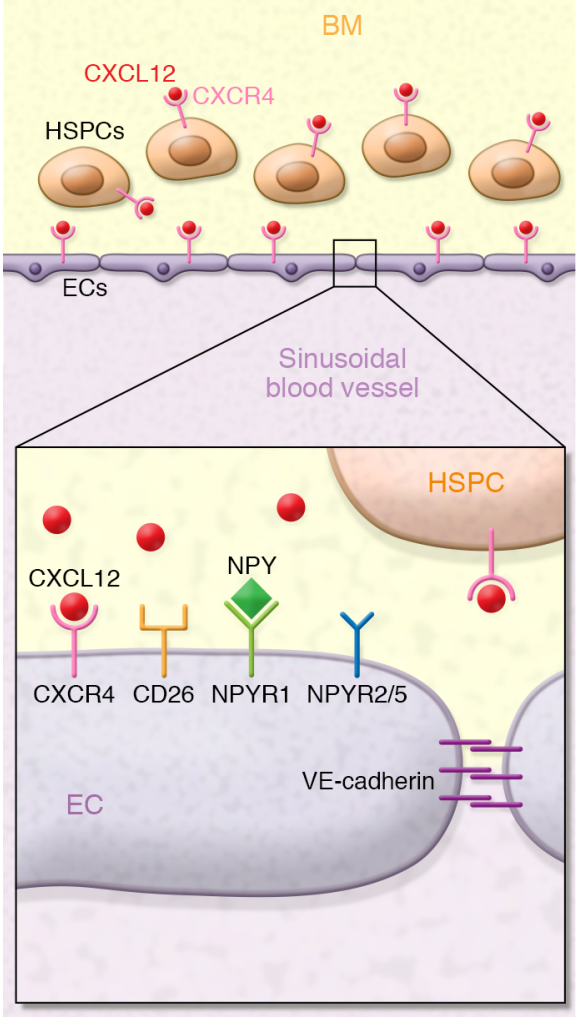

B G-CSF stimulation (WT)

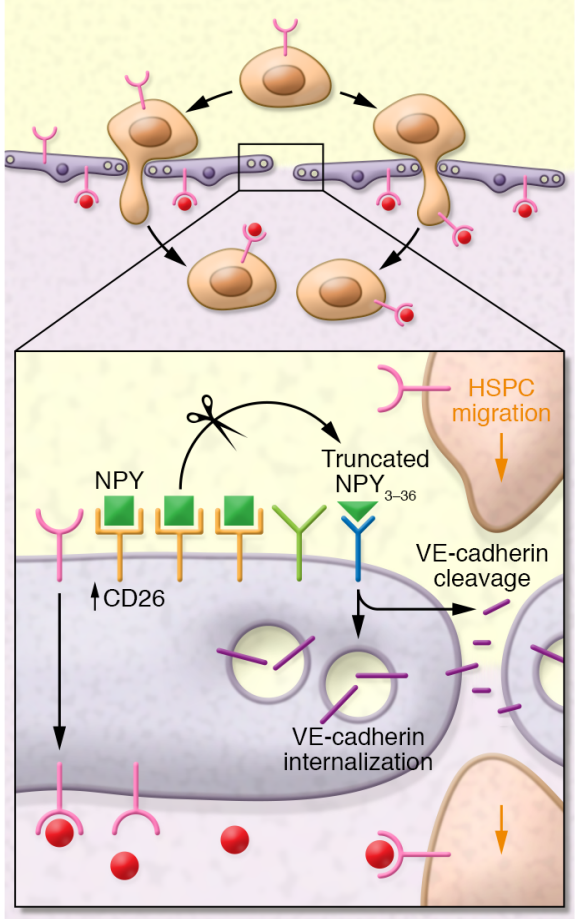

C G-CSF stimulation (CD26-KO)

Figure 1. Model of the mechanisms that promote G-CSF-induced HSPC mobilization. G-CSF stimulation activates the redelivery of CXCL12 (red) from the BM to the vessel lumen by the endothelium-expressed CXCR4 receptor (pink), thereby favoring mobilization of HSPCs to the periphery. In parallel, CD26 (yellow) levels are increased on the ECs, promoting NPY cleavage into its truncated form (NPY ${ }_{3-36}$, dark green), which in turn binds with higher affinity to NPYR2/5 (blue). Stimulation of NPY receptors triggers VE-cadherin (purple) internalization and degradation, enhancing BM vascular permeability. Increasing the permeability of the vascular barrier further promotes HSPC activation by enhancing intracellular HSPC ROS levels, HSPC motility, and the

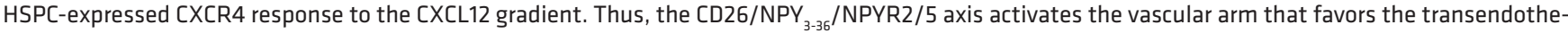
lial migration of HSPCs into the peripheral blood. In the absence of CD26, NPY cryptic domain truncation is impaired, diminishing NPYR2/5 activation. As a consequence, in CD26-deficient mice, the vascular barrier retains the same steady-state permeability properties, preventing the full activation and translocation of BM HSPCs into the sinusoidal lumen.

activity by the truncated cryptic forms of these proteins. Despite an increase in the binding affinity of truncated CSF proteins for their receptors (19), the truncated domains exhibited reduced CSF activity and negatively regulate hematopoiesis under stressinduced conditions (18), suggesting a possible regulatory negative feedback role for CD26 during G-CSF-induced mobilization. Additionally, CD26 is expressed by endothelial cells (ECs), and inhibition of CD26 maintains proper vascular barrier function during stress-induced conditions (20).

\section{$B M$ vasculature regulation of HSPC trafficking}

Previous work demonstrated that trafficking of mature and immature hematopoietic cells is regulated by BM endothelial cells (BMECs) (21), suggesting the involvement of BMECs in successful BM transplantation procedures (22). ECs are first responders to stimulatory agents directing HSPC mobilization, as they express the sensory receptors for major participants in the mobilization process, including CXCR 4 and $\beta$-adrenergic receptors (refs. 7, 8, and Figure 1).

BMECs respond to stimulus by actively relocating CXCL12 molecules from the abluminal side (BM parenchyma) to the lumen, which is in contact with the blood circulation $(7,8)$. A recent study ascertained a pivotal role for BM vascular permeability in regulating the activation and induction of HSPC motility (23).

All in vivo trafficking events of mature and immature hematopoietic cells are restricted primarily to the highly permeable sinusoidal blood vessels. Moreover, genetic or pharmacological disruption of proper vascular barrier function increases blood vessel permeability, boosts HSPC trafficking, and mobilizes HSPCs to the peripheral blood. Mechanistically, enhanced vascular fenestration allows higher penetration of blood plasma-carried factors, which in turn augment ROS levels in HSPCs (23). Enhanced intracellular ROS instigate HSPC motility and trafficking in response to CXCL12. Pharmacological scavenging of ROS restores normal HSPC levels in the peripheral blood and normalizes the in vitro migration levels of HSPCs toward CXCL12 (23).

It is now established that the permeability of the blood-BM barrier impacts $\mathrm{BM}$ homeostasis and dictates HSPC fate decisions. However, the factors that influence the permeability of the blood$\mathrm{BM}$ barrier during stress-induced HSPC mobilization and regulate angiocrine stress response elements, such as sympathetic nervous system- derived neurotransmitters, are unknown. 


\section{Vascular neurocrine signals enhance blood-BM barrier permeability}

In this issue, Singh et al. set out to decipher the mechanism by which CD26 relays G-CSF-induced HSPC mobilization (16, 17). This was achieved by performing sophisticated experiments to determine the extent to which CD26 expression by hematopoietic cells or by stromal cells in the $\mathrm{BM}$ microenvironment is essential for the stem cell mobilization process (1). Contrary to the prior hypothesis suggesting that CXCL12 cleavage by hematopoietic CD26 promotes HSPC migration out of the BM, Singh and colleagues showed that hematopoietic expression of CD26 is not essential for HSPC mobilization. Moreover, CD26 expression was reduced in mobilized HSPCs, and deletion of CD26 in murine HSPCs did not alter HSPC mobilization in a WT environment (1). These unexpected results align with recent reports indicating that CD26 truncates inflammatory cytokines into a nonactive form $(18,19)$ and, as such, may interfere with the hematopoietic response during alert and stress conditions. However, CD26 expression by niche cells was essential for proper HSPC mobilization (Figure 1).

Singh et al. observed that neither inhibition nor genetic deletion of CD26 had an effect on the properties of cells from any of the distinct subtypes of BM mesenchymal stromal cell lineages. Furthermore, ablation of CD26 activity failed to prevent a G-CSF-induced decrease in CXCL12 levels (1). Therefore, CD26 was not one of the in vivo enzymes responsible for CXCL12 degradation in the $\mathrm{BM}$ microenvironment.

Next, Singh and colleagues evaluated CD26 in the context of blood vessel ECs, which form a mechanical barrier between blood circulation and the inner marrow and regulate both $\mathrm{BM}$ stem cell homeostasis and hematopoietic trafficking (23). CD26 expression was upregulated in a subtype of sinusoidal ECs, which represent an exclusive site for HSPC trafficking (23). Encouraged by evidence that CD26 supplied by endothelium promotes hematopoietic transendothelial migration in vitro, Singh et al. screened for a protein target for CD26 and identified the neurotransmitter neuropeptide $\mathrm{Y}$ (NPY) as a promising candidate for modulating HSPC mobilization (1). Like norepinephrine, NPY is a stress response element derived from the sympathetic nervous system and serves as a vasoconstrictor that orchestrates body metabolism and affects neuronal-mediated circadian rhythms (24).

Singh et al. confirmed that NPY was functionally truncated by CD26; moreover, the truncated form $\left(\mathrm{NPY}_{3-36}\right)$ could enhance hematopoietic transendothelial migration. Sinusoidal ECs expressed the NPY receptors 2 and 5, which preferentially bound the truncated form of NPY, and administration of truncated NPY restored normal HSPC mobilization in CD26-deficient mice. In support of this proposition, truncated NPY also rescued the attenuated G-CSF-induced HSPC mobilization observed in $\mathrm{NPY}^{-/-}$mice (Figure 1 and ref. 1). Finally, truncated NPY augmented endothelial barrier permeability by downregulating adherence junction molecules, such as VE-cadherin and CD31. Enhanced sinusoidal permeability was determined by a gap widening between BM vascular ECs that resulted in greater penetration of blood-borne molecules into the BM parenchyma (1).

\section{Conclusions}

Singh et al. provide important insight into the complex mechanism that regulates stem cell mobilization by molecularly eavesdropping on the crosstalk among blood, bone, and brain elements (1). Previous studies have shown the importance of the CXCL12/CXCR4 axis in regulating HSPC mobilization as the immediate response to alarm or stress (2). Singh et al. mechanistically extended these observations by showing that this axis regulates only one arm of HSPC mobilization and requires the activation of additional arms to be effective. Induction of numerous early response factors during hematopoietic recovery facilitates the release of mobilizing factors from the sympathetic nervous system. Notably, norepinephrine promotes the activation of HSPCs and their release from the $\mathrm{BM}$ niche and augments enhanced motility via the CXCL12/ CXCR4 axis. Yet, CD26 enables the activation of the truncated NPY ${ }_{3-36} / \mathrm{NPYR} 2 / 5$ axis that further augments the trafficking of stimulated HSPCs through the primed BM sinusoidal portals. Further studies are warranted to uncover the connection between the neuronal system and vascular regulation and may involve examining the effect of the circadian tone over sinusoidal vascular portals, determining the precise source of NPY, and understanding the dynamic expression of CD26 and NPY receptors on distinct $\mathrm{BM}$ vascular beds in steady-state and stress hematopoiesis. The work by Singh et al. has opened the door for designing new, safer, and effective approaches to induce mobilization of HSPCs for curative therapies in patients who are refractory to current treatments.

\section{Acknowledgments}

The authors are supported by the Ansary Stem Cell Institute, the Starr Foundation Tri-Institutional Stem Cell Initiative, the Empire State Stem Cell Board, and New York State Department of Health grants, and by NIH R01 grants (DK095039, HL119872, HL128158, and HL115128) and U54 CA163167.

Address correspondence to: Shahin Rafii or Tomer Itkin, Weill Cornell Medicine, Department of Medicine, Division of Regenerative Medicine, Ansary Stem Cell Institute, 1300 York Avenue, New York, New York 10065, USA. Phone: 212.746.2070; Email: srafii@med.cornell. edu (S. Rafii). Phone: 212.746.4538; Email: toi2003@med.cornell.edu (T. Itkin).

1. Singh P, et al. Neuropeptide Y regulates a vascular gateway for hematopoietic stem and progenitor cells. JClin Invest.2017;127(12):4527-4540.

2. Lapid K, Glait-Santar C, Gur-Cohen S, Canaani J, Kollet O, Lapidot T. Egress and mobilization of hematopoietic stem and progenitor cells: a dynamic multi-facet process. In: StemBook. Cambridge, Massachusetts, USA: Harvard Stem Cell Institute; 2008

3. Jillella AP, Ustun C. What is the optimum number of $\mathrm{CD}_{34}{ }^{+}$peripheral blood stem cells for an autologous transplant? Stem Cells Dev. 2004;13(6):598-606.

4. Pusic I, et al. Impact of mobilization and remobilization strategies on achieving sufficient stem cell yields for autologous transplantation. Biol Blood Marrow Transplant. 2008;14(9):1045-1056.

5. Lapidot T, Petit I. Current understanding of stem cell mobilization: the roles of chemokines, proteolytic enzymes, adhesion molecules, cytokines, and stromal cells. Exp Hematol. 2002;30(9):973-981.

6. Petit I, et al. G-CSF induces stem cell mobilization by decreasing bone marrow SDF-1 and up-regulating CXCR4. Nat Immunol. 2002;3(7):687-694.

7. Dar A, et al. Rapid mobilization of hematopoietic progenitors by AMD3100 and catecholamines is mediated by CXCR4-dependent SDF-1 release from bone marrow stromal cells. Leukemia. 
2011;25(8):1286-1296.

8. Dar A, et al. Chemokine receptor CXCR4dependent internalization and resecretion of functional chemokine SDF-1 by bone marrow endothelial and stromal cells. Nat Immunol. 2005;6(10):1038-1046.

9. Méndez-Ferrer S, Lucas D, Battista M, Frenette PS. Haematopoietic stem cell release is regulated by circadian oscillations. Nature. 2008;452(7186):442-447.

10. Spiegel A, et al. Catecholaminergic neurotransmitters regulate migration and repopulation of immature human CD $34^{+}$cells through Wnt signaling. Nat Immunol. 2007;8(10):1123-1131.

11. Katayama Y, et al. Signals from the sympathetic nervous system regulate hematopoietic stem cell egress from bone marrow. Cell. 2006;124(2):407-421.

12. Lucas D, et al. Norepinephrine reuptake inhibition promotes mobilization in mice: potential impact to rescue low stem cell yields. Blood. 2012;119(17):3962-3965.

13. Heissig B, et al. Recruitment of stem and progenitor cells from the bone marrow niche requires
MMP-9 mediated release of kit-ligand. Cell. 2002;109(5):625-637.

14. Vagima Y, et al. MT1-MMP and RECK are involved in human $\mathrm{CD}_{3} 4^{+}$progenitor cell retention, egress, and mobilization. J Clin Invest. 2009;119(3):492-503.

15. Kollet O, et al. Osteoclasts degrade endosteal components and promote mobilization of hematopoietic progenitor cells. Nat Med. 2006;12(6):657-664.

16. Christopherson KW, Cooper S, Broxmeyer HE. Cell surface peptidase CD26/DPPIV mediates G-CSF mobilization of mouse progenitor cells. Blood. 2003;101(12):4680-4686.

17. Christopherson KW, Cooper S, Hangoc G, Broxmeyer HE. CD26 is essential for normal G-CSF-induced progenitor cell mobilization as determined by CD26-/ mice. Exp Hematol. 2003;31(11):1126-1134.

18. Broxmeyer HE, et al. Dipeptidylpeptidase 4 negatively regulates colony-stimulating factor activity and stress hematopoiesis. Nat Med. 2012;18(12):1786-1796.

19. O'Leary HA, et al. DPP4 truncated GM-CSF and IL-3 manifest distinct receptor-binding and regulatory functions compared with their full-length forms. Leukemia. 2017;31(11):2468-2478.

20. Hashimoto N, et al. DPP-4 inhibition protects human umbilical vein endothelial cells from hypoxia-induced vascular barrier impairment. JPharmacol Sci. 2017;135(1):29-36.

21. Möhle R, Moore MA, Nachman RL, Rafii S. Transendothelial migration of $\mathrm{CD} 34^{+}$and mature hematopoietic cells: an in vitro study using a human bone marrow endothelial cell line. Blood. 1997;89(1):72-80.

22. Rafii S, Mohle R, Shapiro F, Frey BM, Moore MA. Regulation of hematopoiesis by microvascular endothelium. Leuk Lymphoma. 1997;27(5-6):375-386.

23. Itkin $\mathrm{T}$, et al. Distinct bone marrow blood vessels differentially regulate haematopoiesis. Nature. 2016;532(7599):323-328.

24. Kuo LE, et al. Neuropeptide Y acts directly in the periphery on fat tissue and mediates stressinduced obesity and metabolic syndrome. Nat Med. 2007;13(7):803-811. 